\title{
Euler's Approximations of Weak Solutions of Reflecting SDEs with Discontinuous Coefficients
}

\author{
by \\ Alina SEMRAU \\ Presented by Jerzy $Z A B C Z Y K$
}

Summary. We study convergence in law for the Euler and Euler-Peano schemes for stochastic differential equations reflecting on the boundary of a general convex domain. We assume that the coefficients are measurable and continuous almost everywhere with respect to the Lebesgue measure. The proofs are based on new estimates of Krylov's type for the approximations considered.

1. Introduction. Consider a $d$-dimensional stochastic differential equation (SDE) on a convex domain $D$ with reflecting boundary condition

$$
X_{t}=X_{0}+\int_{0}^{t} \sigma\left(X_{s}\right) d W_{s}+\int_{0}^{t} b\left(X_{s}\right) d s+K_{t}, \quad t \in \mathbb{R}^{+} .
$$

Here $X_{0}=x_{0} \in \bar{D}=D \cup \partial D, X$ is a reflecting process on $\bar{D}, K$ is a bounded variation process with variation $|K|$ increasing only when $X_{t} \in \partial D, W$ is a $d$-dimensional standard Wiener process and $\sigma: \bar{D} \rightarrow \mathbb{R}^{d} \otimes \mathbb{R}^{d}$ and $b: \bar{D} \rightarrow \mathbb{R}^{d}$ are measurable functions continuous almost everywhere with respect to the Lebesgue measure, i.e.

$$
l\left(D_{\sigma \sigma^{*}}\right)=0, \quad l\left(D_{b}\right)=0,
$$

where $D_{\sigma \sigma^{*}}, D_{b}$ are the sets of discontinuity points of $\sigma \sigma^{*}$ and $b$ respectively. We assume that $\sigma$ and $b$ satisfy two additional conditions:

$$
\begin{aligned}
& \left\|\sigma \sigma^{*}(x)\right\|+|b(x)|^{2} \leq L\left(1+|x|^{2}\right), \quad x \in \mathbb{R}^{d}, \\
& \left(\sigma \sigma^{*}(y) x, x\right) \geq \lambda|x|^{2}, \quad x, y \in \mathbb{R}^{d},
\end{aligned}
$$

for some constants $L, \lambda>0$.

2000 Mathematics Subject Classification: 60H20, 60H99, 60F17.

Key words and phrases: stochastic differential equation, reflecting boundary condition, Skorokhod problem. 
In this paper we investigate two approximations of $X$, discrete $\left\{\bar{X}^{n}\right\}$ and continuous $\left\{X^{n}\right\}$, defined to be the solutions of the SDEs with reflecting boundary conditions of the form

$$
\bar{X}_{t}^{n}=X_{0}+\int_{0}^{t} \sigma\left(\bar{X}_{s-}^{n}\right) d W_{s}^{\varrho^{n}}+\int_{0}^{t} b\left(\bar{X}_{s-}^{n}\right) d \varrho_{s}^{n}+\bar{K}_{t}^{n}, \quad t \in \mathbb{R}^{+},
$$

and

$$
X_{t}^{n}=X_{0}+\int_{0}^{t} \sigma\left(X_{s-}^{n, \varrho^{n}}\right) d W_{s}+\int_{0}^{t} b\left(X_{s-}^{n, \varrho^{n}}\right) d s+K_{t}^{n}, \quad t \in \mathbb{R}^{+},
$$

respectively, where $\varrho_{t}^{n}=\max \{k / n: k \in \mathbb{N} \cup\{0\}, k / n \leq t\}$ and $W_{t}^{\varrho^{n}}$ is the discretization of $W$, i.e. $W_{t}^{\varrho^{n}}=W_{k / n}$ for $t \in[k / n,(k+1) / n), k \in \mathbb{N} \cup\{0\}$, $n \in \mathbb{N}$. Since in the case $D=\mathbb{R}^{d}$ the processes $\left\{\bar{X}^{n}\right\},\left\{X^{n}\right\}$ are the classical Euler and Euler-Peano approximations to the equation without reflecting boundary, we call them the Euler and Euler-Peano approximations to (1.1). Such approximations have been considered before when the coefficients $\sigma, b$ of (1.1) are continuous and the SDE (1.1) has the pathwise uniqueness property (see e.g. Słomiński $(1994,2001)$ ). In this paper we omit the assumption of the continuity of $\sigma, b$. We give two new theorems concerning convergence in law of $\left\{\bar{X}^{n}\right\}$ and $\left\{X^{n}\right\}$ to a solution of (1.1) in which the coefficients satisfy the conditions (1.2)-(1.4) and under the assumption that (1.1) has a unique weak solution.

Conditions ensuring weak uniqueness for (1.1) in the case of discontinuous coefficients $\sigma, b$ were considered in Stroock and Varadhan (1971) and Schmidt (1989). In the latter paper it is shown that if $d=1$ and $b \equiv 0$, then (1.1) has a weak solution on $\bar{D}=\left[r_{1}, r_{2}\right]$ for every starting point $x_{0} \in \bar{D}$ iff the set $M$ of all $x \in \bar{D}$ such that $\int_{\bar{D} \cap U_{x}} \sigma^{-2}(y) d y=\infty$ for every open neighbourhood $U_{x}$ of $x$ is contained in the set $N$ of zeros of $\sigma$. Therefore, if $\sigma$ is merely bounded and measurable, some additional assumptions on boundedness of $\left(\sigma \sigma^{*}\right)^{-1}$ are indispensable. Schmidt also proved that in the above situation the solution of (1.1) is unique if $N \subseteq M$. In the multidimensional case, from Stroock and Varadhan (1971) we know that a sufficient condition for the uniqueness of a weak solution of (1.1) is that $\sigma \sigma^{*}$ is bounded, continuous and uniformly elliptic, $b$ is bounded measurable and $\partial D$ is regular. Under these assumptions the uniqueness can be shown with the use of the Girsanov theorem.

In Yan (2002) a similar problem is considered. In the case of nonreflecting SDEs Yan shows that the convergence of the Euler-Peano scheme to the solution of (1.1) holds as long as a weak solution exists and is unique. He assumes that $\sigma$ and $b$ are continuous almost everywhere with respect to the Lebesgue measure and have at most linear growth. However, since he 
uses the local time technique in his proofs, the main results are only onedimensional. In our paper, the main tool is a version of the multidimensional Krylov inequality.

Let us now introduce some definitions and notations. $\mathcal{D}\left(\mathbb{R}^{+}, \mathbb{R}^{d}\right)$ is the space of all mappings $x: \mathbb{R}^{+} \rightarrow \mathbb{R}^{d}$ which are right continuous and admit left-hand limits, with the Skorokhod topology $J_{1}$. Processes we consider have trajectories in $\mathcal{D}\left(\mathbb{R}^{+}, \mathbb{R}^{d}\right)$. For a given process $X$ we write $\Delta X_{t}$ for $X_{t}-X_{t-}$ and we denote by $X^{\varrho^{n}}$ the discretization of $X$, i.e. $X_{t}^{\varrho^{n}}=X_{k / n}$ for $t \in$ $[k / n,(k+1) / n), k \in \mathbb{N} \cup\{0\}, n \in \mathbb{N}$. If $X=\left(X^{1}, \ldots, X^{d}\right)$ is a local martingale then $[X]_{t}$ stands for $\sum_{i=1}^{d}\left[X^{i}\right]_{t}$, where $\left[X^{i}\right]$ is the quadratic variation process of $X^{i}, i=1, \ldots, d$. If $K=\left(K^{1}, \ldots, K^{d}\right)$ is a process with locally finite variation then $|K|_{t}=\sum_{i=1}^{d}\left|K^{i}\right|_{t}$, where $\left|K^{i}\right|_{t}$ is the total variation of $K^{i}$ on $[0, t] . L^{p}(Q), p \geq 1$, is the usual $L^{p}$-space with the Lebesgue measure $l$ on $Q . \mathbb{R}^{d} \otimes \mathbb{R}^{d}$ is the space of $d \times d$-matrices with the norm $\|\sigma\|=\left(\operatorname{tr} \sigma \sigma^{*}\right)^{1 / 2}$ and $\sigma^{*}$ is the matrix transpose to $\sigma$. We write $K_{R}^{d}=\left\{x \in \mathbb{R}^{d}:|x| \leq R\right\}$, where $|\cdot|$ denotes the usual Euclidean norm on $\mathbb{R}^{d}$. Finally, " $\rightarrow \mathcal{D}$ " and " $\rightarrow \mathcal{P}$ " denote convergence in law and in probability respectively.

2. The Euler scheme. Let $D$ be a convex domain in $\mathbb{R}^{d}$. Define the set $\mathcal{N}_{x}$ of inward normal unit vectors at $x \in \partial D$ by

$$
\mathcal{N}_{x}=\left\{n \in \mathbb{R}^{d}:|n|=1, \bigwedge_{y \in \bar{D}}\langle y-x, n\rangle \geq 0\right\} .
$$

Let $(\Omega, \mathcal{F}, P)$ be a probability space and $\left(\mathcal{F}_{t}\right)_{t \in \mathbb{R}^{+}}$be a filtration on $(\Omega, \mathcal{F}, P)$ satisfying the usual conditions. Let $Y$ be an $\left(\mathcal{F}_{t}\right)$-adapted semimartingale with initial value in $\bar{D}$, i.e.

$$
Y_{t}=Y_{0}+M_{t}+A_{t}, \quad t \in \mathbb{R}^{+},
$$

where $Y_{0} \in \bar{D}, M$ is an $\left(\mathcal{F}_{t}\right)$-adapted local martingale, $A$ is an $\left(\mathcal{F}_{t}\right)$-adapted process with bounded variation, $M_{0}=A_{0}=0$. Recall that a pair $(X, K)$ of $\left(\mathcal{F}_{t}\right)$-adapted processes is called a solution to the Skorokhod problem associated with $Y$ if:

(i) $X_{t}=Y_{t}+K_{t}, t \in \mathbb{R}^{+}$,

(ii) $X$ is $\bar{D}$-valued,

(iii) $K$ is a process with locally bounded variation such that $K_{0}=0$ and

$$
K_{t}=\int_{0}^{t} n_{s} d|K|_{s}, \quad|K|_{t}=\int_{0}^{t} \mathbf{1}_{\left\{X_{s} \in \partial D\right\}} d|K|_{s}, \quad t \in \mathbb{R}^{+},
$$

where $n_{s} \in \mathcal{N}_{X_{s}}$ if $X_{s} \in \partial D$.

Recall also that the SDE (1.1) is said to have a weak solution if there exists a filtered probability space $\left(\bar{\Omega}, \overline{\mathcal{F}},\left(\overline{\mathcal{F}}_{t}\right), \bar{P}\right)$, an $\left(\overline{\mathcal{F}}_{t}\right)$-adapted Wiener 
process $\bar{W}$ and a pair of $\left(\overline{\mathcal{F}}_{t}\right)$-adapted processes $(\bar{X}, \bar{K})$ such that $(\bar{X}, \bar{K})$ is a solution of the Skorokhod problem associated with

$$
\bar{Y}_{t}=\bar{X}_{0}+\int_{0}^{t} \sigma\left(\bar{X}_{s}\right) d \bar{W}_{s}+\int_{0}^{t} b\left(\bar{X}_{s}\right) d s .
$$

If for any two weak solutions $(X, K, W),\left(\Omega, \mathcal{F},\left(\mathcal{F}_{t}\right), P\right)$ and $(\bar{X}, \bar{K}, \bar{W})$, $\left(\bar{\Omega}, \overline{\mathcal{F}},\left(\overline{\mathcal{F}}_{t}\right), \bar{P}\right)$ with the same initial distribution, the laws of $(X, K)$ and $(\bar{X}, \bar{K})$ are the same then we say that weak uniqueness holds for (1.1).

Let $\left(\mathcal{F}_{t}^{\varrho^{n}}\right)$ denote the discretization of $\left(\mathcal{F}_{t}\right)$, i.e. $\mathcal{F}_{t}^{\varrho^{n}}=\mathcal{F}_{k / n}$ for $t \in$ $[k / n,(k+1) / n), \quad k \in \mathbb{N} \cup\{0\}, n \in \mathbb{N}$. We say that the $\operatorname{SDE}(1.5)$ has a strong solution if there exists a pair $\left(\bar{X}^{n}, \bar{K}^{n}\right)$ of $\left(\overline{\mathcal{F}}_{t}^{\varrho^{n}}\right)$-adapted processes such that $\left(\bar{X}^{n}, \bar{K}^{n}\right)$ is a solution of the Skorokhod problem associated with

$$
\bar{Y}_{t}^{n}=X_{0}+\int_{0}^{t} \sigma\left(\bar{X}_{s-}^{n}\right) d W_{s}^{\varrho^{n}}+\int_{0}^{t} b\left(\bar{X}_{s-}^{n}\right) d \varrho_{s}^{n} .
$$

It is easy to prove that a solution $\bar{X}^{n}$ of (1.5) is given by the recurrent formula

$$
\begin{aligned}
\bar{X}_{0}^{n} & =X_{0}, \\
\bar{X}_{(k+1) / n}^{n} & =\Pi\left(\bar{X}_{k / n}^{n}+b\left(\bar{X}_{k / n}^{n}\right) \frac{1}{n}+\sigma\left(\bar{X}_{k / n}^{n}\right)\left(W_{(k+1) / n}-W_{k / n}\right)\right)
\end{aligned}
$$

and $\bar{X}_{t}^{n}=\bar{X}_{k / n}^{n}$ for $t \in[k / n,(k+1) / n), k \in \mathbb{N} \cup\{0\}, n \in \mathbb{N}$, where $\Pi(x)$ is a projection of $x$ on $\bar{D}$.

We can now formulate our main result.

TheOREM 2.1. Let $\left\{\left(\bar{X}^{n}, \bar{K}^{n}\right)\right\}_{n \in \mathbb{N}}$ be a sequence of solutions of (1.5) with coefficients $\sigma, b$ satisfying (1.2)-(1.4). If (1.1) has a unique weak solution $(X, K)$ then $\left(\bar{X}^{n}, \bar{K}^{n}\right) \rightarrow_{\mathcal{D}}(X, K)$.

Proof. Our proof starts with the observation that $\left\{\sup _{t \leq T}\left|\bar{X}_{t}^{n}\right|\right\}_{n \in \mathbb{N}}$ and $\left\{\left|\bar{K}^{n}\right|_{T}\right\}_{n \in \mathbb{N}}$ are bounded in probability, i.e.

$$
\begin{aligned}
& \lim _{R \rightarrow \infty} \limsup _{n \rightarrow \infty} P\left(\sup _{t \leq T}\left|\bar{X}_{t}^{n}\right| \geq R\right)=0, \quad T \in \mathbb{R}^{+}, \\
& \lim _{R \rightarrow \infty} \limsup _{n \rightarrow \infty} P\left(\left|\bar{K}^{n}\right|_{T} \geq R\right)=0, \quad T \in \mathbb{R}^{+} .
\end{aligned}
$$

Since the pair $\left(\bar{X}^{n}, \bar{K}^{n}\right)$ is a solution of the Skorokhod problem associated with $\bar{Y}^{n}$ given by the formula (2.1), due to Corollary 2.6 in Słomiński (2001), for every $p \in \mathbb{N}$ and every $q \in \mathbb{R}^{+}$,

$$
\sup _{n \in \mathbb{N}} E \sup _{t \leq q}\left|\bar{X}_{t}^{n}\right|^{2 p}<\infty, \quad \sup _{n \in \mathbb{N}} E\left|\bar{K}^{n}\right|_{q}^{p}<\infty,
$$

which in particular implies boundedness in probability of our sequences. 
Now we prove that $\left\{\left(\bar{X}^{n}, \bar{K}^{n}, W^{\varrho^{n}}\right)\right\}_{n \in \mathbb{N}}$ is tight in $\mathcal{D}\left(\mathbb{R}^{+}, \mathbb{R}^{3 d}\right)$. We use the criterion given by Aldous (1978). Let $\left\{\gamma_{n}\right\}_{n \in \mathbb{N}}$ be a sequence of $\left(\mathcal{F}_{t}^{\varrho^{n}}\right)$ stopping times and let $\left\{\delta_{n}\right\}_{n \in \mathbb{N}}$ be a sequence of positive constants such that $\delta_{n} \downarrow 0$ and $\gamma_{n}+\delta_{n} \leq q, n \in \mathbb{N}, q \in \mathbb{R}^{+}$. By Corollary 1 in Słomiński (1994), (1.3) and (2.2),

$$
\begin{aligned}
E\left|\bar{X}_{\gamma_{n}+\delta_{n}}^{n}-\bar{X}_{\gamma_{n}}^{n}\right|^{2} & \leq C_{1} E\left(\left[\int_{0}^{\cdot} \sigma\left(\bar{X}_{s-}^{n}\right) d W_{s}^{\varrho^{n}}\right]_{\gamma_{n}}^{\gamma_{n}+\delta_{n}}+\left|\int_{\gamma_{n}}^{\gamma_{n}+\delta_{n}} b\left(\bar{X}_{s-}^{n}\right) d \varrho_{s}^{n}\right|_{\gamma_{n}}^{2}\right) \\
& \leq C_{2} E\left(\int_{\gamma_{n}}^{\gamma_{n}+\delta_{n}} \operatorname{tr} \sigma \sigma^{*}\left(\bar{X}_{s-}^{n}\right) d \varrho_{s}^{n}+\int_{\gamma_{n}}^{\gamma_{n}+\delta_{n}}\left|b\left(\bar{X}_{s-}^{n}\right)\right|^{2} d \varrho_{s}^{n}\right) \\
& \leq L C_{2}(d+1) E \int_{\gamma_{n}}^{\gamma_{n}+\delta_{n}}\left(1+\sup _{u \leq s}\left|\bar{X}_{u}^{n}\right|^{2}\right) d s \\
& =L C_{3}(d+1) \delta_{n} \underset{n \rightarrow \infty}{\longrightarrow} 0 .
\end{aligned}
$$

Using again Corollary 1 in Słomiński (1994), (1.3) and (2.2) we obtain the same estimate for $E\left|\bar{K}_{\gamma_{n}+\delta_{n}}^{n}-\bar{K}_{\gamma_{n}}^{n}\right|^{2}$, and consequently, the convergence

$$
E\left|\bar{K}_{\gamma_{n}+\delta_{n}}^{n}-\bar{K}_{\gamma_{n}}^{n}\right|^{2} \underset{n \rightarrow \infty}{\longrightarrow} 0 \text {. }
$$

Since $W^{\varrho^{n}} \rightarrow_{\mathcal{D}} W$, it follows that the sequence $\left\{\left(\bar{X}^{n}, \bar{K}^{n}, W^{\varrho^{n}}\right)\right\}_{n \in \mathbb{N}}$ is tight in $\mathcal{D}\left(\mathbb{R}^{+}, \mathbb{R}^{3 d}\right)$. Therefore, there exists a subsequence $\left(n^{\prime}\right) \subset(n)$ and processes $\bar{X}, \bar{K}, \bar{W}$ such that

$$
\left(\bar{X}^{n^{\prime}}, \bar{K}^{n^{\prime}}, W^{\varrho^{n^{\prime}}}\right) \underset{\mathcal{D}}{\longrightarrow}(\bar{X}, \bar{K}, \bar{W})
$$

in $\mathcal{D}\left(\mathbb{R}^{+}, \mathbb{R}^{3 d}\right)$, where $\bar{W}$ is a Wiener process with respect to the natural filtration $\mathcal{F}^{\bar{X}}, \bar{K}, \bar{W}$. By the standard argument, in order to finish the proof it is sufficient to show that $(\bar{X}, \bar{K})$ is a solution of the SDE

$$
\bar{X}_{t}=\bar{X}_{0}+\int_{0}^{t} \sigma\left(\bar{X}_{s}\right) d \bar{W}_{s}+\int_{0}^{t} b\left(\bar{X}_{s}\right) d s+\bar{K}_{t}, \quad t \in \mathbb{R}^{+} .
$$

Now we prove an inequality of Krylov's type for $\left\{\bar{X}^{n}\right\}_{n \in \mathbb{N}}$, which is our crucial tool.

Lemma 2.2. Let $\tau_{n}^{R}=\inf \left\{t:\left|\bar{X}_{t}^{n}\right|>R\right\}, R \in \mathbb{R}^{+}, n \in \mathbb{N}$. Then for all bounded measurable functions $f: \bar{D} \rightarrow \mathbb{R}^{+}$such that $l\left(D_{f}\right)=0$,

$$
\limsup _{n \rightarrow \infty} E \int_{0}^{T \wedge \tau_{n}^{R}} f\left(\bar{X}_{s-}^{n}\right) d s \leq C\|f\|_{L^{d}\left(K_{R}^{d} \cap D\right)}
$$

where $C$ is a constant depending only on $d, T, R$ and $\lambda$. 
Proof. For every $n \in \mathbb{N}$ set

$$
\widehat{Y}_{t}^{n}=X_{0}+\int_{0}^{t} \sigma\left(\bar{X}_{s-}^{n}\right) d W_{s}+\int_{0}^{t} b\left(\bar{X}_{s-}^{n}\right) d s
$$

and let $\left(\widehat{X}^{n}, \widehat{K}^{n}\right)$ be a solution of the Skorokhod problem associated with $\widehat{Y}^{n}$. In view of Corollary 2.6 in Słomiński (2001), for every $p \in \mathbb{N}$ and every $q \in \mathbb{R}^{+}$,

$$
\sup _{n \in \mathbb{N}} E\left|\widehat{K}^{n}\right|_{q}^{p}<\infty .
$$

Let $\omega_{W}(\delta, g)$ denote the modulus of continuity of $W$. By Schwarz's inequality, Lemma A.4 in Słomiński (2001), (1.3) and (2.2),

$$
\begin{aligned}
E \sup _{t \leq q}\left|\widehat{Y}_{t}^{n}-\bar{Y}_{t}^{n}\right|^{2}=E \sup _{t \leq q}\left|\sigma\left(\bar{X}_{\varrho_{t}^{n}}^{n}\right)\left(W_{t}-W_{\varrho_{t}^{n}}\right)+b\left(\bar{X}_{\varrho_{t}^{n}-}^{n}\right)\left(t-\varrho_{t}^{n}\right)\right|^{2} \\
\leq 2\left\{d\left(E \sup _{t \leq q}\left\|\sigma \sigma^{*}\left(\bar{X}_{t-}^{n}\right)\right\|^{2}\right)^{1 / 2}\left(E\left\{\omega_{W}(1 / n, q)\right\}^{4}\right)^{1 / 2}\right. \\
\left.+\left(\frac{1}{n}\right)^{2} E \sup _{t \leq q}\left|b\left(\bar{X}_{t-}^{n}\right)\right|^{2}\right\} \\
\leq C_{1}\left\{\frac{\ln n}{n}\left(E \sup _{t \leq q}\left(1+\left|\bar{X}_{t-}^{n}\right|^{4}\right)\right)^{1 / 2}+\left(\frac{1}{n}\right)^{2} E \sup _{t \leq q}\left(1+\left|\bar{X}_{t-}^{n}\right|^{2}\right)\right\} \\
\leq C_{2} \frac{\ln n}{n} .
\end{aligned}
$$

Applying Lemma 2.2 in Tanaka (1979) we conclude that

$$
\sup _{t \leq q}\left|\widehat{X}_{t}^{n}-\bar{X}_{t}^{n}\right|^{2} \leq \sup _{t \leq q}\left|\widehat{Y}_{t}^{n}-\bar{Y}_{t}^{n}\right|^{2}+4 \sup _{t \leq q}\left|\widehat{Y}_{t}^{n}-\bar{Y}_{t}^{n}\right|\left(\left|\widehat{K}^{n}\right|_{q}-\left|\bar{K}^{n}\right|_{q}\right) .
$$

Hence and by Schwarz's inequality,

$$
\begin{aligned}
E \sup _{t \leq q}\left|\widehat{X}_{t}^{n}-\bar{X}_{t}^{n}\right|^{2} \leq & C_{3}\left\{E \sup _{t \leq q}\left|\widehat{Y}_{t}^{n}-\bar{Y}_{t}^{n}\right|^{2}\right. \\
& \left.+\left(E \sup _{t \leq q}\left|\widehat{Y}_{t}^{n}-\bar{Y}_{t}^{n}\right|^{2}\right)^{1 / 2}\left(E\left|\widehat{K}^{n}\right|_{q}^{2}+E\left|\bar{K}^{n}\right|_{q}^{2}\right)^{1 / 2}\right\} .
\end{aligned}
$$

Using now (2.2), (2.6) and the above estimate of $E \sup _{t \leq q}\left|\widehat{Y}_{t}^{n}-\bar{Y}_{t}^{n}\right|^{2}$ we get

$$
E \sup _{t \leq q}\left|\widehat{X}_{t}^{n}-\bar{X}_{t}^{n}\right|^{2} \leq C_{4}\left(\frac{\ln n}{n}\right)^{1 / 2}
$$

By the assumption made at the beginning of the proof, $\widehat{X}^{n}$ is of the form

$$
\widehat{X}_{t}^{n}=X_{0}+\widehat{A}_{t}^{n}+\widehat{M}_{t}^{n}+\widehat{K}_{t}^{n},
$$

where $\widehat{A}_{t}^{n}=\int_{0}^{t} b\left(\bar{X}_{s-}^{n}\right) d s$ and $\widehat{M}_{t}^{n}=\int_{0}^{t} \sigma\left(\bar{X}_{s-}^{n}\right) d W_{s}$. For every $n \in \mathbb{N}$ let $\widehat{\tau}_{n}^{R^{\prime}}=\inf \left\{t:\left|\widehat{X}_{t}^{n}\right|>R^{\prime}\right\}$, where $R^{\prime} \in \mathbb{R}^{+}$and $R^{\prime}>R$. According to (2.7), 
we have

$$
\lim _{n \rightarrow \infty} P\left(\tau_{n}^{R}>\widehat{\tau}_{n}^{R^{\prime}}\right)=0 .
$$

It is easy to note that $E\left|\widehat{A}^{n}\right|_{T \wedge \widehat{\tau}_{n}^{R^{\prime}}}<\infty$ for every $n \in \mathbb{N}$. From (2.6) we also obtain $E\left|\widehat{K}^{n}\right|_{T \wedge \widehat{\tau}_{n}^{R^{\prime}}}<\infty$ for every $n \in \mathbb{N}$. Moreover, by (1.4), the matrix $Q$ defined as

$$
Q_{t}=\left(\frac{\left(\sigma \sigma^{*}\right)^{i j}\left(\bar{X}_{t-}^{n}\right) d t}{\operatorname{tr} \sigma \sigma^{*}\left(\bar{X}_{t-}^{n}\right) d t}\right)_{i, j=1, \ldots, d}
$$

is uniformly elliptic. All the assumptions of Theorem 6(i) in Melnikov (1983) are satisfied, so there exists a constant $C_{5}$ depending only on $d, T, R$ and $\lambda$ such that

$$
E \int_{0}^{T \wedge \widehat{\tau}_{n}^{R^{\prime}}} f\left(\widehat{X}_{s}^{n}\right) d\left\langle\widehat{M}^{n}\right\rangle_{s} \leq C_{5}\|f\|_{L^{d}\left(K_{R^{\prime}}^{d} \cap D\right)} .
$$

On the other hand,

$$
\begin{aligned}
E \int_{0}^{T \wedge \widehat{\tau}_{n}^{R^{\prime}}} f\left(\widehat{X}_{s}^{n}\right) d s & \leq(d \lambda)^{-1} E \int_{0}^{T \wedge \widehat{\tau}_{n}^{R^{\prime}}} f\left(\widehat{X}_{s}^{n}\right) \operatorname{tr} \sigma \sigma^{*}\left(\bar{X}_{s-}^{n}\right) d s \\
& =(d \lambda)^{-1} E \int_{0}^{T \wedge \widehat{\tau}_{n}^{R^{\prime}}} f\left(\widehat{X}_{s}^{n}\right) d\left\langle\widehat{M}^{n}\right\rangle_{s},
\end{aligned}
$$

so for all measurable functions $f: \bar{D} \rightarrow \mathbb{R}^{+}$and for every $n \in \mathbb{N}$,

$$
E \int_{0}^{T \wedge \widehat{\tau}_{n}^{R^{\prime}}} f\left(\widehat{X}_{s}^{n}\right) d s \leq C\|f\|_{L^{d}\left(K_{R^{\prime}}^{d} \cap D\right)},
$$

where $C$ is a constant depending only on $d, T, R^{\prime}$ and $\lambda$.

We are now able to show that

$$
\limsup _{n \rightarrow \infty} E \int_{0}^{T \wedge \tau_{n}^{R}} f\left(\widehat{X}_{s}^{n}\right) d s \leq C\|f\|_{L^{d}\left(K_{R}^{d} \cap D\right)} .
$$

Indeed, from the property of $\widehat{\tau}_{n}^{R^{\prime}}$ and (2.8),

$$
\begin{array}{r}
\limsup _{n \rightarrow \infty} E \int_{0}^{T \wedge \tau_{n}^{R}} f\left(\widehat{X}_{s}^{n}\right) d s \leq \limsup _{n \rightarrow \infty} E \int_{0}^{T \wedge \widehat{\tau}_{n}^{R^{\prime}}} f\left(\widehat{X}_{s}^{n}\right) d s \mathbf{1}_{\left(\tau_{n}^{R} \leq \widehat{\tau}_{n}^{R^{\prime}}\right)} \\
+\limsup _{n \rightarrow \infty} E \int_{0}^{T \wedge \tau_{n}^{R}} f\left(\widehat{X}_{s}^{n}\right) d s \mathbf{1}_{\left(\tau_{n}^{R}>\widehat{\tau}_{n}^{R^{\prime}}\right)}
\end{array}
$$




$$
\leq \limsup _{n \rightarrow \infty} E \int_{0}^{T \wedge \widehat{\tau}_{n}^{R^{\prime}}} f\left(\widehat{X}_{s}^{n}\right) d s+C_{6} \limsup _{n \rightarrow \infty} P\left(\tau_{n}^{R}>\widehat{\tau}_{n}^{R^{\prime}}\right) \leq C\|f\|_{L^{d}\left(K_{R^{\prime}}^{d} \cap D\right)} .
$$

Letting $R^{\prime} \downarrow R$ yields (2.9).

Next, we show that for every continuous function $f: \bar{D} \rightarrow \mathbb{R}^{+}$,

$$
\limsup _{n \rightarrow \infty} E\left|\int_{0}^{T \wedge \tau_{n}^{R}} f\left(\widehat{X}_{s-}^{n}\right) d s-\int_{0}^{T \wedge \tau_{n}^{R}} f\left(\bar{X}_{s-}^{n}\right) d s\right|=0 .
$$

Let $M:=\sup _{x \in K_{R}^{d} \cap \bar{D}}|f(x)|<\infty$. Since $f$ is uniformly continuous on $K_{R}^{d} \cap \bar{D}$, for every $\varepsilon>0$ there exists $\delta>0$ such that $\left|f(x)-f\left(x^{\prime}\right)\right|<\varepsilon$ if $x, x^{\prime} \in K_{R}^{d} \cap \bar{D}$ and $\left|x-x^{\prime}\right|<\delta$. Therefore, by Chebyshev's inequality,

$$
\begin{aligned}
E \mid \int_{0}^{T \wedge \tau_{n}^{R}}\left(f\left(\widehat{X}_{s-}^{n}\right)-\right. & \left.f\left(\bar{X}_{s-}^{n}\right)\right) d s \mid \\
\leq & E \int_{0}^{T \wedge \tau_{n}^{R}}\left|f\left(\widehat{X}_{s-}^{n}\right)-f\left(\bar{X}_{s-}^{n}\right)\right| \mathbf{1}_{\left(\left|\widehat{X}_{s-}^{n}-\bar{X}_{s-}^{n}\right|<\delta\right)} d s \\
& +E \int_{0}^{T \wedge \tau_{n}^{R}}\left(f\left(\widehat{X}_{s-}^{n}\right)-f\left(\bar{X}_{s-}^{n}\right)\right) \mathbf{1}_{\left(\left|\widehat{X}_{s-}^{n}-\bar{X}_{s-}^{n}\right| \geq \delta\right)} d s \\
\leq & \varepsilon T+2 M T P\left(\sup _{s \leq T \wedge \tau_{n}^{R}}\left|\widehat{X}_{s-}^{n}-\bar{X}_{s-}^{n}\right| \geq \delta\right) \\
\leq & \varepsilon T+2 M T \delta^{-2} E \sup _{t \leq T \wedge \tau_{n}^{R}}\left|\widehat{X}_{t}^{n}-\bar{X}_{t}^{n}\right|^{2} .
\end{aligned}
$$

Using (2.7) and letting $\varepsilon \rightarrow 0$ we obtain (2.10). Hence, by (2.9), it follows that the inequality (2.5) is true for every continuous function $f: \bar{D} \rightarrow \mathbb{R}^{+}$.

Now let $f$ be an arbitrary nonnegative bounded function such that $l\left(D_{f}\right)$ $=0$. Since $f$ is Riemann integrable, for every $\varepsilon>0$ there exists a continuous function $f_{\varepsilon}^{+}$such that $f \leq f_{\varepsilon}^{+}$and

$$
\int_{K_{R}^{d} \cap D}\left(f_{\varepsilon}^{+}(x)-f(x)\right) d x<\varepsilon .
$$

Since for $f_{\varepsilon}^{+}$the inequality (2.5) is true,

$$
\begin{aligned}
\limsup _{n \rightarrow \infty} E \int_{0}^{T \wedge \tau_{n}^{R}} f\left(\bar{X}_{s-}^{n}\right) d s & \leq \limsup _{n \rightarrow \infty} E \int_{0}^{T \wedge \tau_{n}^{R}} f_{\varepsilon}^{+}\left(\bar{X}_{s-}^{n}\right) d s \\
& \leq C\left\|f_{\varepsilon}^{+}\right\|_{L^{d}\left(K_{R}^{d} \cap D\right)} \leq C\|f\|_{L^{d}\left(K_{R}^{d} \cap D\right)}+C_{7} \varepsilon^{1 / d} .
\end{aligned}
$$

Letting $\varepsilon \rightarrow 0$ completes the proof of the lemma. 
The remaining part of the proof consists in a modification of the proof of Theorem 2.2 from Rozkosz and Słomiński (1997). For every $n \in \mathbb{N}$ let $\bar{\tau}_{n}^{R}=\inf \left\{t \in \mathbb{R}^{+}:\left|\bar{X}_{t}^{n}\right| \geq R\right.$ or $\left.\left|\bar{X}_{t-}^{n}\right| \geq R\right\}$ and $\bar{\tau}^{R}=\inf \left\{t \in \mathbb{R}^{+}:\left|\bar{X}_{t}\right| \geq R\right.$ or $\left.\left|\bar{X}_{t-}\right| \geq R\right\}, R \in \mathbb{R}^{+}$. In view of (2.3) and Proposition VI.2.12 from Jacod and Shiryaev (2003), there exists a sequence $\left\{R_{k}\right\}_{k \in \mathbb{N}}$ with $R_{k} \uparrow \infty$ such that for every $k \in \mathbb{N}$,

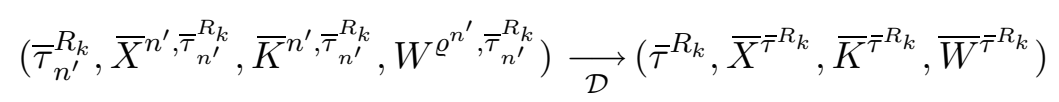

in $\mathbb{R} \times \mathcal{D}\left(\mathbb{R}^{+}, \mathbb{R}^{3 d}\right)$.

Using mollification we can construct sequences $\left\{b_{i}\right\}_{i \in \mathbb{N}}$ and $\left\{\sigma_{i}\right\}_{i \in \mathbb{N}}$ of continuous functions $\sigma_{i}: \bar{D} \rightarrow \mathbb{R}^{d} \otimes \mathbb{R}^{d}, b_{i}: \bar{D} \rightarrow \mathbb{R}^{d}$ such that $\left\|\sigma_{i} \sigma_{i}^{*}(x)\right\|+$ $\left|b_{i}(x)\right|^{2} \leq K\left(1+|x|^{2}\right), x \in \mathbb{R}^{d}$, and $\sigma_{i}^{k, j} \rightarrow \sigma^{k, j}$ and $b_{i}^{l} \rightarrow b^{l}$ in $L^{d}(K)$, $k, j, l=1, \ldots, d$, for every compact subset $K \subset \bar{D}$. By (2.3) and Theorem 2.6 of Jakubowski, Mémin and Pages (1989), it follows that for every $i \in \mathbb{N}, k \in \mathbb{N}$,

$$
\begin{gathered}
\left(\bar{\tau}_{n^{\prime}}^{R_{k}}, \bar{X}^{n^{\prime}, \bar{\tau}_{n^{\prime}}^{R_{k}}}, \int_{0}^{\cdot \wedge \bar{\tau}_{n_{k}^{\prime}}^{R_{k}}} \sigma_{i}\left(\bar{X}_{s-}^{n^{\prime}}\right) d W_{s}^{\varrho_{s}^{n^{\prime}}}, \int_{0}^{\cdot \wedge \bar{\tau}_{n^{\prime}}^{R_{k}}} b_{i}\left(\bar{X}_{s-}^{n^{\prime}}\right) d \varrho_{s}^{n^{\prime}}, K^{n^{\prime}, \bar{\tau}_{n^{\prime}}^{R_{k}}}\right) \\
\underset{\mathcal{D}}{\longrightarrow}\left(\bar{\tau}^{R_{k}}, \bar{X}^{\bar{\tau}^{R_{k}}}, \int_{0}^{\wedge \bar{\tau}^{R_{k}}} \sigma_{i}\left(\bar{X}_{s}\right) d \bar{W}_{s}, \int_{0}^{\cdot \wedge \bar{\tau}_{k}^{R_{k}}} b_{i}\left(\bar{X}_{s}\right) d s, \bar{K}^{\tau^{R_{k}}}\right)
\end{gathered}
$$

in $\mathbb{R} \times \mathcal{D}\left(\mathbb{R}^{+}, \mathbb{R}^{4 d}\right)$.

On the other hand, by Lemma 2.2 and the Lebesgue dominated convergence theorem, for every $k \in \mathbb{N}$ and every $T \in \mathbb{R}^{+}$,

$$
\begin{aligned}
& \lim _{i \rightarrow \infty} \limsup _{n^{\prime} \rightarrow \infty} E\left[\int_{0}\left(\sigma_{i}-\sigma\right)\left(\bar{X}_{s-}^{n^{\prime}}\right) d W_{s}^{\varrho^{n^{\prime}}}\right] \\
&=\lim _{i \rightarrow \infty} \limsup _{n^{\prime} \rightarrow \infty} E \int_{0}^{T \wedge \bar{\tau}_{n^{\prime}}^{R_{k}}} \int_{0}^{R_{k}} \\
& \leq \lim _{i \rightarrow \infty} \limsup _{n^{\prime} \rightarrow \infty} E \int_{0}^{T \wedge \bar{\tau}^{R_{k}}} \operatorname{tr}\left(\sigma_{i}-\sigma\right)\left(\sigma_{i}-\sigma\right)^{*}\left(\bar{X}_{s-}^{n^{\prime}}\right) d \varrho_{s}^{n^{\prime}} \\
& \leq C \lim _{i \rightarrow \infty}\|\|\left(\sigma_{i}-\sigma\right)\left(\sigma_{i}-\sigma\right)^{*}\left\|^{2}\right\|_{L^{d}\left(K_{R_{k}}^{d} \cap D\right)}=0 .
\end{aligned}
$$

Similarly, 


$$
\begin{aligned}
& \lim _{i \rightarrow \infty} \limsup _{n^{\prime} \rightarrow \infty} E \mid \int_{0}\left(b_{i}-b\right)\left.\left(\bar{X}_{s-}^{n^{\prime}}\right) d \varrho_{s}^{n^{\prime}}\right|_{T \wedge \bar{\tau}_{n^{\prime}}^{R_{k}}} \\
& \leq d^{1 / 2} \lim _{i \rightarrow \infty} \limsup _{n^{\prime} \rightarrow \infty} E \int_{0}^{T \wedge \bar{\tau}_{n^{\prime}}^{R_{k}}}\left|\left(b_{i}-b\right)\left(\bar{X}_{s-}^{n^{\prime}}\right)\right| d s \\
& \leq d^{1 / 2} C \lim _{i \rightarrow \infty}\left\|\left|b_{i}-b\right|\right\|_{L^{d}\left(K_{R_{k}}^{d} \cap D\right)}=0 .
\end{aligned}
$$

Boundedness in probability of $\left\{\sup _{t \leq T}\left|X_{t}^{n}\right|\right\}_{n \in \mathbb{N}}$ implies that for every $T$ in $\mathbb{R}^{+}$we have $\lim _{k \rightarrow \infty} \lim \sup _{n^{\prime} \rightarrow \infty} P\left(\bar{\tau}_{n^{\prime}}^{R_{k}} \leq T\right)=0$. Hence, for every $\varepsilon>0$,

$$
\lim _{i \rightarrow \infty} \limsup _{n^{\prime} \rightarrow \infty} P\left(\sup _{t \leq T}\left|\int_{0}^{t} \sigma_{i}\left(\bar{X}_{s-}^{n^{\prime}}\right) d W_{s}^{\varrho^{n^{\prime}}}-\int_{0}^{t} \sigma\left(\bar{X}_{s-}^{n^{\prime}}\right) d W_{s}^{\varrho^{n^{\prime}}}\right| \geq \varepsilon\right)=0
$$

and

$$
\lim _{i \rightarrow \infty} \limsup _{n^{\prime} \rightarrow \infty} P\left(\sup _{t \leq T}\left|\int_{0}^{t} b_{i}\left(\bar{X}_{s-}^{n^{\prime}}\right) d \varrho_{s}^{n^{\prime}}-\int_{0}^{t} b\left(\bar{X}_{s-}^{n^{\prime}}\right) d \varrho_{s}^{n^{\prime}}\right| \geq \varepsilon\right)=0 .
$$

Now we show that Krylov's inequality is also true for $\bar{X}$. First assume that $f$ is continuous. By (2.11) and Theorem 2.6 of Jakubowski, Mémin and Pages (1989),

$$
\int_{0}^{\cdot \wedge \bar{\tau}_{n^{\prime}}^{R_{k}}} f\left(\bar{X}_{s-}^{n^{\prime}}\right) d s \underset{\mathcal{D}}{\longrightarrow} \int_{0}^{\cdot \wedge \bar{\tau}^{R_{k}}} f\left(\bar{X}_{s}\right) d s \quad \text { in } \mathcal{D}\left(\mathbb{R}^{+}, \mathbb{R}^{d}\right) .
$$

Therefore, by Lemma 2.2 and Fatou's lemma, for every $k \in \mathbb{N}$ and $T \in \mathbb{R}^{+}$,

$$
E \int_{0}^{T \wedge \bar{\tau}^{R_{k}}} f\left(\bar{X}_{s}\right) d s \leq \liminf _{n^{\prime} \rightarrow \infty} E \int_{0}^{T \wedge \bar{\tau}_{n^{\prime}}^{R_{k}}} f\left(\bar{X}_{s-}^{n^{\prime}}\right) d s \leq C\|f\|_{L^{d}\left(K_{R}^{d} \cap D\right)} .
$$

The proof of this inequality for all nonnegative measurable functions $f$ is now standard (see e.g. Krylov (1982)).

By the above, in much the same way as for $\bar{X}^{n^{\prime}}$ and $W^{\varrho^{n^{\prime}}}$, we show that for every $T \in \mathbb{R}^{+}$,

$$
\lim _{i \rightarrow \infty} E\left\langle\int_{0}^{\dot{0}}\left(\sigma_{i}-\sigma\right)\left(\bar{X}_{s}\right) d \bar{W}_{s}\right\rangle_{T \wedge \bar{\tau}^{R_{k}}}=0
$$

and

$$
\lim _{i \rightarrow \infty} E\left|\int_{0}\left(b_{i}-b\right)(\bar{X}) d s\right|_{T \wedge \bar{\tau}^{R_{k}}}=0
$$


Since $\bar{\tau}^{R_{k}} \uparrow \infty P$-a.s., for every $\varepsilon>0$,

$$
\lim _{i \rightarrow \infty} P\left(\sup _{t \leq T}\left|\int_{0}^{t} \sigma_{i}\left(\bar{X}_{s}\right) d \bar{W}_{s}-\int_{0}^{t} \sigma\left(\bar{X}_{s}\right) d \bar{W}_{s}\right| \geq \varepsilon\right)=0
$$

and

$$
\lim _{i \rightarrow \infty} P\left(\sup _{t \leq T}\left|\int_{0}^{t} b_{i}\left(\bar{X}_{s}\right) d s-\int_{0}^{t} b\left(\bar{X}_{s}\right) d s\right| \geq \varepsilon\right)=0 .
$$

By (2.12)-(2.16) and Theorem 3.2 from Billingsley (1999),

$$
\begin{aligned}
&\left(\bar{X}^{n^{\prime}}, \int_{0}^{1} \sigma\left(\bar{X}_{s-}^{n^{\prime}}\right) d W_{s}^{\varrho_{s}^{n^{\prime}}}, \int_{0}^{1} b\left(\bar{X}_{s-}^{n^{\prime}}\right) d \varrho_{s}^{n^{\prime}}, K^{n^{\prime}}\right) \\
& \underset{\mathcal{D}}{\longrightarrow}\left(\bar{X}, \int_{0} \sigma\left(\bar{X}_{s}\right) d \bar{W}_{s}, \int_{0} b\left(\bar{X}_{s}\right) d s, \bar{K}\right)
\end{aligned}
$$

in $\mathcal{D}\left(\mathbb{R}^{+}, \mathbb{R}^{4 d}\right)$. Finally, by the continuous mapping theorem, $(\bar{X}, \bar{K})$ is a solution of the SDE (2.4), which completes the proof.

3. The Euler-Peano scheme. Let us now consider a continuous approximation $\left\{X^{n}\right\}$ of $X$ defined as the solutions of the SDEs (1.6). For that approximation one can obtain a similar result to that for $\left\{\bar{X}^{n}\right\}$. We describe it in the following theorem.

TheOREM 3.1. Let $\left\{\left(X^{n}, K^{n}\right)\right\}_{n \in \mathbb{N}}$ be a sequence of solutions of (1.6) with coefficients $\sigma, b$ satisfying (1.2)-(1.4). If (1.1) has a unique weak solution $(X, K)$ then $\left(X^{n}, K^{n}\right) \rightarrow_{\mathcal{D}}(X, K)$.

The proof of this theorem is quite similar to that of Theorem 2.1 because Lemma 2.2 holds true with $\bar{X}^{n}$ replaced by $X^{n, \varrho^{n}}$.

The result given in Theorem 3.1 is quite satisfactory from the theoretical standpoint. However, if we are interested in numerical simulation there appears a problem - not always can one give a simple simulating scheme of a solution of (1.6). In the case $\bar{D}=\mathbb{R}^{d-1} \times \mathbb{R}^{+}$an efficient method of such simulation was given by Lépingle (1995).

Acknowledgements. I would like to thank Professor Leszek Słomiński for suggesting the problem and for many stimulating conversations.

\section{References}

D. J. Aldous (1978), Stopping time and tightness, Ann. Probab. 6, 335-340.

P. Billingsley (1999), Convergence of Probability Measures, Wiley, New York.

J. Jacod and A. N. Shiryaev (2003), Limit Theorems for Stochastic Processes, Springer, Berlin. 
A. Jakubowski, J. Mémin et G. Pages (1989), Convergence en loi des suites d'intégrales stochastiques sur l'espace $D^{1}$ de Skorokhod, Probab. Theory Related Fields 81, 111137.

N. V. Krylov (1982), Controlled Diffusion Processes, Springer, New York.

D. Lépingle (1995), Euler scheme for reflected stochastic differential equations, Math. Comput. Simulation 38, 119-126.

A. V. Melnikov (1983), Stochastic equations and Krylov's estimates for semimartingales, Stochastics 10, 81-102.

R. Pettersson (1995), Approximations for stochastic differential equations with reflecting convex boundaries, Stochastic Process. Appl. 59, 295-308.

A. Rozkosz and L. Słomiński (1997), On stability and existence of solutions of SDEs with reflection at the boundary, Stochastic Process. Appl. 68, 285-302.

W. Schmidt (1989), On stochastic differential equations with reflecting barriers, Math. Nachr. 142, 135-148.

L. Słomiński (1994), On approximation of solutions of multidimensional SDEs with reflecting boundary conditions, Stochastic Process. Appl. 50, 197-219.

L. Słomiński (1996), Stability of stochastic differential equations driven by general semimartingales, Dissertationes Math. 349.

L. Słomiński (2001), Euler's approximations of solutions of SDEs with reflecting boundary, Stochastic Process. Appl. 94, 317-337.

D. W. Stroock and S. R. S. Varadhan (1971), Diffusion processes with boundary conditions, Comm. Pure Appl. Math. 24, 147-225.

H. Tanaka (1979), Stochastic differential equations with reflecting boundary condition in convex regions, Hiroshima Math. J. 9, 163-177.

L. Yan (2002), The Euler scheme with irregular coefficients, Ann. Probab. 30, 1172-1194.

Alina Semrau

Institute of Mathematics and Physics

University of Technology and Agriculture

Kaliskiego 7

85-796 Bydgoszcz, Poland

E-mail: alucha@atr.bydgoszcz.pl

Received November 9, 2006;

received in final form March 22, 2007 